Few-Body Systems Suppl. 0, 1-12 (2002)

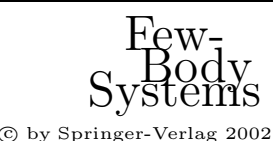

(c) by Springer-Verlag 2002 Printed in Austria

\title{
Three-Nucleon Electroweak Capture Reactions
}

\author{
L.E. Marcucci ${ }^{1,2}$, M. Viviani ${ }^{2,1}$, A. Kievsky ${ }^{2,1}$, S. Rosati ${ }^{1,2}$, R. Schiavilla ${ }^{3,4}$ \\ ${ }^{1}$ Physics Department, University of Pisa, Via Buonarroti 2, I-56100 Pisa, \\ Italy \\ ${ }^{2}$ INFN, Sezione di Pisa, Via Buonarroti 2, I-56100 Pisa, Italia \\ 3 Jefferson Laboratory, Newport News, Virginia 23606, USA \\ ${ }^{4}$ Physics Department, Old Dominion University, Norfolk, VA 23529, USA
}

\begin{abstract}
Recent advances in the study of the $p-d$ radiative and $\mu-{ }^{3}$ He weak capture processes are presented and discussed. The three-nucleon bound and scattering states are obtained using the correlated-hyperspherical-harmonics method, with realistic Hamiltonians consisting of the Argonne $v_{14}$ or Argonne $v_{18}$ two-nucleon and Tucson-Melbourne or Urbana IX three-nucleon interactions. The electromagnetic and weak transition operators include one- and two-body contributions. The theoretical accuracy achieved in these calculations allows for interesting comparisons with experimental data.
\end{abstract}

\section{Introduction}

A number of electromagnetic (EM) and weak transitions in light nuclei have interesting astrophysical implications. They are also very important for an understanding of nuclear structure and dynamics. The theoretical description of these processes requires the knowledge of the initial and final nuclear states, which can be either bound or in the continuum. Furthermore, it is important to use a realistic model for the EM and weak current operators. The threenucleon systems, in particular, provide a unique "laboratory" due to the capability, achieved in the last few years, of obtaining very accurate bound and continuum wave functions. The accuracy of the calculated three-nucleon wave functions has been verified by comparing results for a variety of observables obtained by a number of groups using different techniques [1]. At present, good overall agreement exists between the theoretical and experimental $N-d$ elastic and inelastic scattering observables (a notable exception, however, is the $A_{y}$ analyzing power at low energies) [2, 3]. Therefore the study of EM and weak transitions in the three-nucleon system does not suffer of uncertainties related 
to the computation of the nuclear wave functions and it is a direct test of the nuclear Hamiltonian $H$ from which these wave functions are obtained, and of the model used to describe the nuclear currents. Since the nuclear EM current is related to $H$ through current conservation, it is clear that the two topics are inter-related. Furthermore, it is interesting to understand whether relativistic corrections as well as $\Delta$-isobar and additional sub-nucleonic degrees of freedom play a role in these processes.

The model for the nuclear EM and weak current considered here has been recently reviewed in Refs. 14, 5, 6. and has been tested in numerous few-nucleon processes. It includes one- and two-body operators. The one-body operators are obtained directly from the non-relativistic limit of the covariant single-nucleon vector and axial currents. In the study of the muon capture, the contribution coming from the induced pseudo-scalar term of the nucleon axial current has to be included (it gives a negligible contribution to $\beta$-decay processes). However, the experimental value of the corresponding form factor $G_{P S}\left(q_{\sigma}^{2}\right)$ is rather uncertain. Assuming pion-pole dominance, the partially conserved axial current (PCAC) hypothesis, and the Goldberger-Treiman relation, $G_{P S}$ is predicted to be $17,8,9,10,11$

$$
G_{P S}^{P C A C}\left(q_{\sigma}^{2}\right)=-\frac{2 m_{\mu} m_{N}}{m_{\pi}^{2}+q_{\sigma}^{2}} G_{A}\left(q_{\sigma}^{2}\right)
$$

where $q_{\sigma}$ is the four-momentum transferred to the nuclear system, $m_{N}, m_{\mu}$ and $m_{\pi}$ indicates the nucleon, muon and pion mass, respectively, and $G_{A}$ is the axial form factor. In our calculation, we have assumed

$$
G_{P S}\left(q_{\sigma}^{2}\right)=R_{P S} G_{P S}^{P C A C}\left(q_{\sigma}^{2}\right)
$$

where $R_{P S}$ is a parameter which has been varied to study the sensibility of our results to this form factor and to investigate to which extent $G_{P S}^{\text {expt }}=G_{P S}^{\mathrm{PCAC}}$. However, most of the calculations have been performed with the choice $R_{P S}=$ 1.

The two-body EM current is separated in two terms. There is a "modelindependent" part which is constructed consistently with the nucleon-nucleon interaction, in order to satisfy the current conservation relation 12 . Current conservation is only approximate for the momentum dependent terms. The second part includes "model-dependent" contributions which come from the $\rho \pi \gamma$ and $\omega \pi \gamma$ processes and the $\Delta$ degrees of freedom. The latter contribution is included in the current and in the nuclear medium in an approximate way, by following the procedure described in Ref. [13. The two-body weak vector current is then obtained from the isovector part of the EM current, in accordance with the conserved-vector-current hypothesis.

Two-body terms have been taken into account in both the axial charge and current operators. The two-body axial charge operator has been obtained consistently with the two-nucleon interaction model, following the methods of Ref. [14. The two-body axial current operators are derived from a mesonexchange model, including $\pi$ - and $\rho$-exchanges and the $\rho \pi$-transition processes, 
as well as $\Delta$-isobar excitation [7, 15]. The latter process gives the dominant contribution. However, its magnitude depends critically on the value adopted for the $N \Delta$ axial coupling constant $g_{A}^{*}$. In the quark model, $g_{A}^{*}$ is related in a simple way to the axial coupling constant of the nucleon $g_{A}\left(g_{A} \approx 1.26\right)$. However, given the uncertainties inherent in quark model predictions, a more reliable estimate for $g_{A}^{*}$ is obtained by adjusting its value to reproduce the experimental value of the Gamow-Teller matrix element in tritium $\beta$-decay $\sqrt{7}$, 15. In this way, the model dependence of the weak axial current is significantly reduced, as shown by previous studies of proton weak captures on ${ }^{1} \mathrm{H}$ 15 and ${ }^{3} \mathrm{He}[7]$. It is important to note that the value of $g_{A}^{*}$ depends on how the $\Delta$ isobar degrees of freedom are treated. Moreover, given the procedure used to determine $g_{A}^{*}$, the latter cannot be naively interpreted as the $N \Delta$ axial coupling constant, since the contributions of additional resonances not included in the present study will contaminate its value.

The ${ }^{3} \mathrm{He},{ }^{3} \mathrm{H}$ bound and the $p-d$ continuum wave functions have been calculated by expanding on a basis of pair-correlated hyperspherical harmonic $(\mathrm{PHH})$ functions 16]. Such a technique has been proven to be very accurate. Various $n-d$ elastic scattering observables calculated by solving the Faddeev equations [2] are in remarkable agreement with the corresponding results obtained with the PHH technique. For example, the phase shift and mixing angle parameters calculated by the two methods at the center-of-mass (c.m.) energy $E_{\text {c.m. }}=2 \mathrm{MeV}$ have been found to differ at level of $0.1 \%$, at most [1]. It should be pointed out that with the PHH technique the inclusion of the Coulomb potential, clearly very important in the energy range considered here, does not present any difficulty.

This paper is organized as follows. The $p-d$ radiative capture and the muon capture on ${ }^{3} \mathrm{He}$ are discussed in Sect. 2 and 3, respectively. The last section contains a summary and some concluding remarks.

\section{Radiative $p-d$ Capture}

The applications of our formalism for c.m. energies ranging from zero to $2 \mathrm{MeV}$, namely below the deuteron breakup threshold (DBT), were already presented in Refs. [17] and [18]. Recently we have extended the PHH technique, in order to compute also $p-d$ scattering wave functions above the DBT [19]. We can therefore compute $p-d$ capture observables at higher energies than previously published. We present here in Fig. 11 a preliminary study at $E_{\text {c.m. }}=3.33 \mathrm{MeV}$, where high-quality data, including differential cross sections, vector and tensor analyzing powers 20] exist. Results for differential cross sections at $E_{\text {c.m. }}=6.67$ and $9.87 \mathrm{MeV}$ and deuteron tensor analyzing powers at $E_{\text {c.m. }}=5.83 \mathrm{MeV}$ are given in Figs. 2 and 3 respectively. The corresponding experimental data are taken from Refs. 221] and [22].

All the theoretical calculations reported in this section have been obtained using the Argonne $v_{18}$ (AV18) [23] two-nucleon and Urbana IX (UIX) 24 threenucleon interactions. By inspection of Fig. 1, we can conclude that our results 

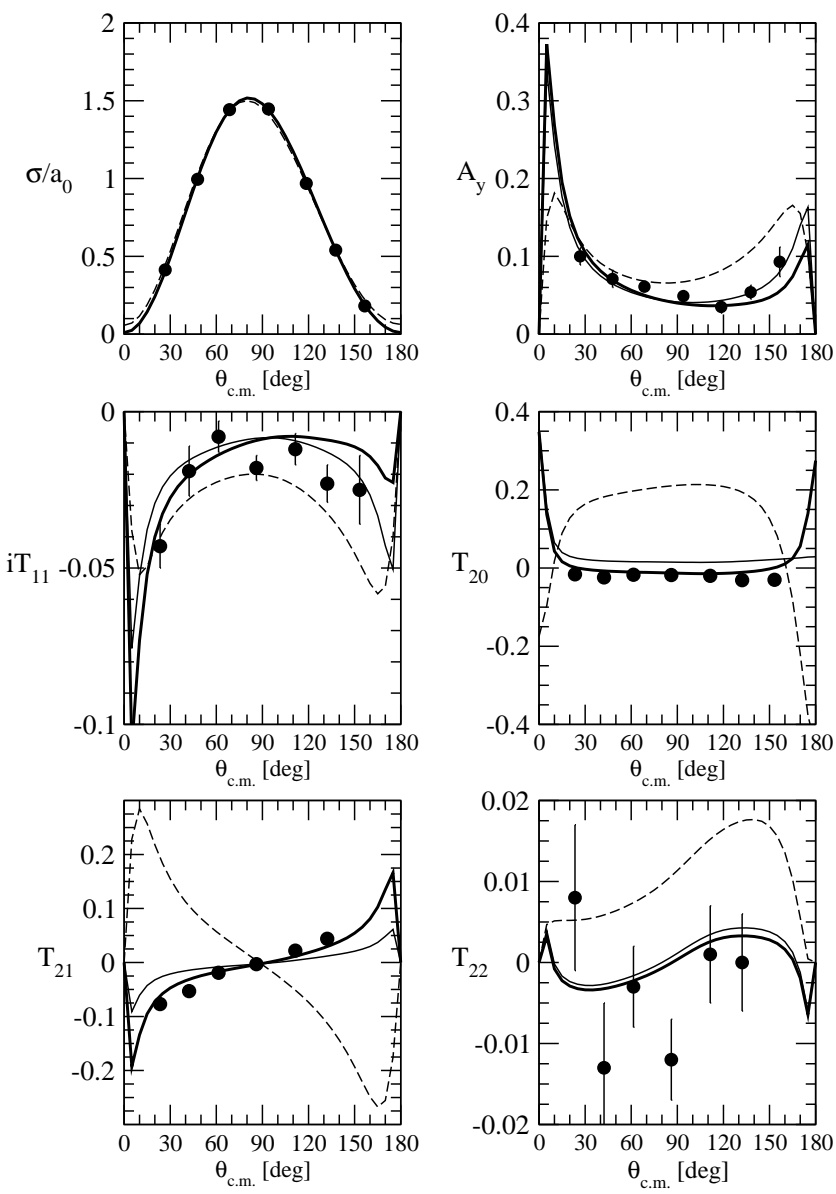

Figure 1. Differential cross section, proton vector analyzing power, and the four deuteron tensor analyzing powers for $p-d$ capture at $E_{\text {c.m. }}=3.33 \mathrm{MeV}$, obtained with the AV18/UIX Hamiltonian model and one-body only (thick dashed lines) or both one- and two-body currents (thin solid lines), are compared with the experimental values of Ref. 20]. The results obtained in the LWA approximation for the spin-flip $E_{1}$ RME's are also shown (thick solid lines). In the first panel, $a_{0}=\int d \Omega \sigma /(4 \pi)$.

(thin solid lines) for the differential cross section and the observables $A_{y}$ and $\mathrm{i} T_{11}$ are in good agreement with the experimental data. On the contrary, for the observables $T_{20}$ and $T_{21}$, significant discrepancies can be observed. A similar situation was found for the $E_{\text {c.m. }}=2 \mathrm{MeV}$ observables 18 . The same situation is present also at higher energies. In fact the differential cross sections in Fig. 2 are well reproduced by our full calculation, as well as the $A_{y}(d)$ observable in Fig. 3, which is basically proportional to i $T_{11}$. Also at $E_{\text {c.m. }}=5.83 \mathrm{MeV}$, as can be seen from Fig. 3, discrepancies are present for the deuteron tensor 

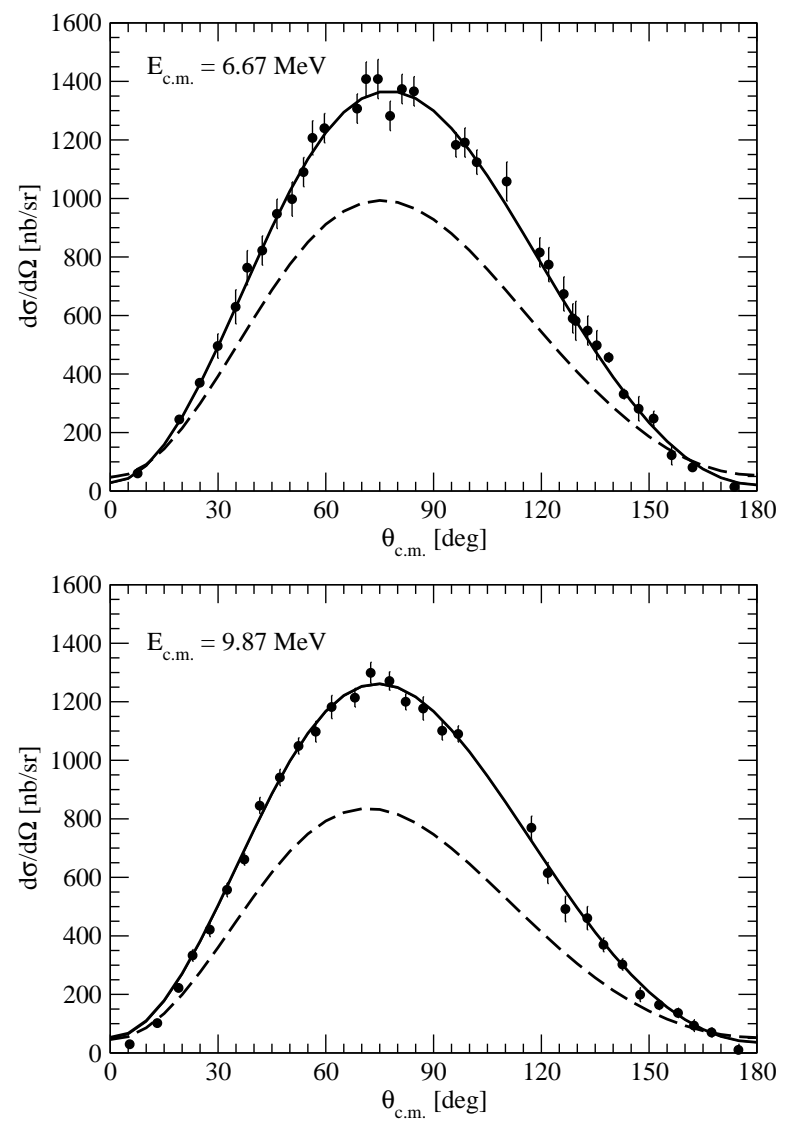

Figure 2. Differential cross section for $p-d$ capture at $E_{\text {c.m. }}=6.67 \mathrm{MeV}$ (top panel) and $E_{\text {c.m. }}=9.87 \mathrm{MeV}$ (lower panel). Notation as in Fig. 1. Thick and thin solid lines are on top of each other. The data are from Ref. 21].

observables $A_{x x}, A_{y y}$ and $A_{z z}$, which are a linear combination of $T_{20}, T_{21}$ and $T_{22}$ (the latter observable is however rather small).

The analysis of the $E_{\text {c.m. }}=2 \mathrm{MeV}$ results in Ref. 18 traced back the problem to an overprediction of the spin-flip electric dipole $E_{1}$ reduced matrix elements (RME's) (namely, those arising from the transitions where the $p-d$ spins in the incident channel are coupled to $S=3 / 2$ ). In fact, when the same RME's are computed in the long wavelength approximation (LWA) at leading order (thick solid line), the observables $T_{20}$ and $T_{21}$ are better reproduced (see Figs. 1 and 3). Interestingly, an analysis of the next-to-leading order terms in the LWA performed in Ref. [18], has shown that they give a sizeable contribution to the spin-flip $E_{1}$ RME's, showing the inadequacy of the use of the leading order only for the calculation of this small spin-flip transition matrix elements. The origin of the discrepancies observed in the deuteron tensor polarization 

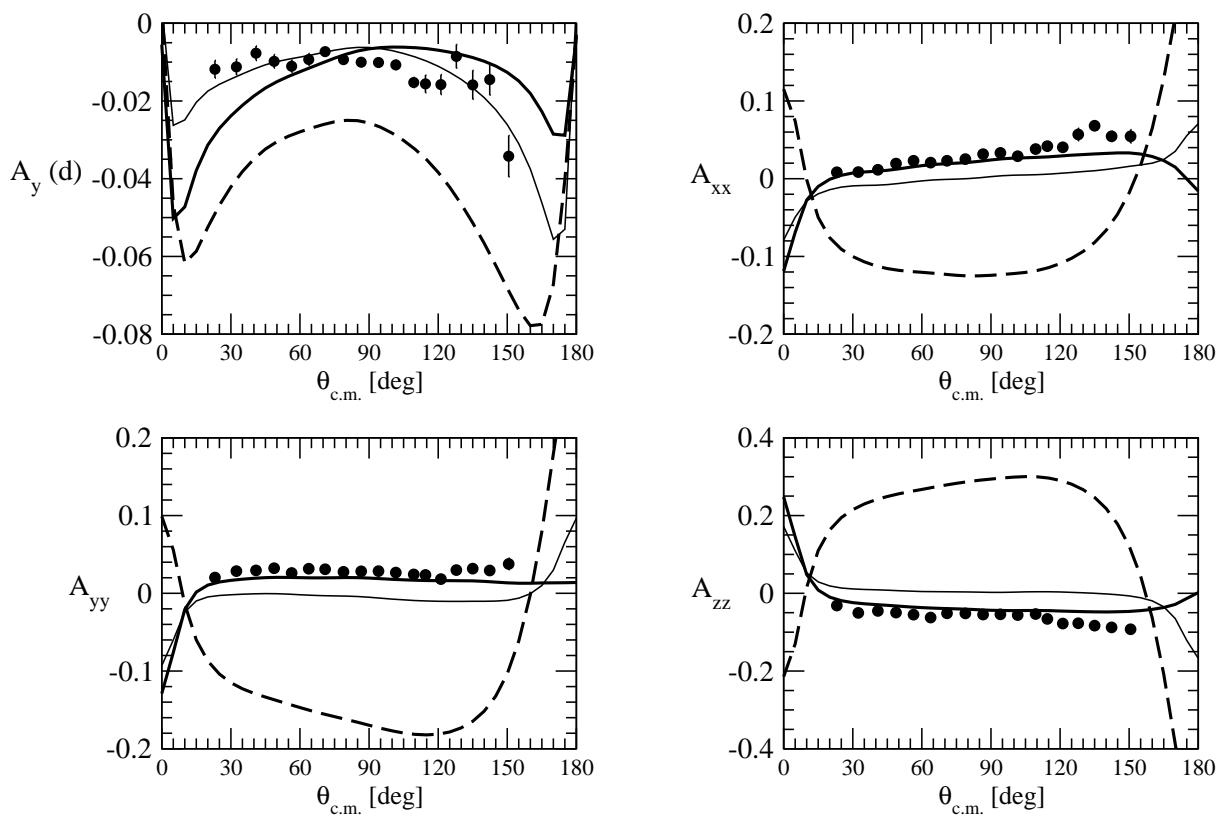

Figure 3. Deuteron tensor analyzing powers for $p-d$ capture at $E_{\text {c.m. }}=5.83 \mathrm{MeV}$. Notation as in Fig. 1. The data are from Ref. [22].

observables is currently under investigation.

\section{Muon Capture}

The $\mu^{-}$weak capture on ${ }^{3} \mathrm{He}$ can occur through three different hadronic channels:

$$
\begin{aligned}
& \mu^{-}+{ }^{3} \mathrm{He} \rightarrow{ }^{3} \mathrm{H}+\nu_{\mu} \quad(70 \%), \\
& \mu^{-}+{ }^{3} \mathrm{He} \rightarrow n+d+\nu_{\mu} \quad(20 \%), \\
& \mu^{-}+{ }^{3} \mathrm{He} \rightarrow n+n+p+\nu_{\mu} \quad(10 \%) \text {. }
\end{aligned}
$$

The focus of the present section is on the first process [8]. Some of the nuclear physics issues in muon capture have been reviewed recently in Ref. [25]. When the triton polarization is not detected, the differential capture rate for the reaction (3) is given by

$$
\frac{\mathrm{d} \Gamma}{\mathrm{d}(\cos \theta)}=\frac{1}{2} \Gamma_{0}\left[1+A_{v} P_{v} \cos \theta+A_{t} P_{t}\left(\frac{3}{2} \cos ^{2} \theta-\frac{1}{2}\right)+A_{\Delta} P_{\Delta}\right],
$$

where $\Gamma_{0}$ is the total capture rate, $A_{v}, A_{t}$ and $A_{\Delta}$ angular correlation parameters and $\theta$ is the angle between the muon polarization and the leptonic 
momentum transfer. The coefficients $P_{v}, P_{t}$ and $P_{\Delta}$ are linear combinations of the probabilities $P\left(f, f_{z}\right)$ of finding the $\mu^{-3}$ He system in the total-spin state $\left|f f_{z}\right\rangle$, and are defined as [26, 27]

$$
\begin{aligned}
P_{v} & =P(1,1)-P(1,-1), \\
P_{t} & =P(1,1)+P(1,-1)-2 P(1,0), \\
P_{\Delta} & =P(1,1)+P(1,-1)+P(1,0)-3 P(0,0) .
\end{aligned}
$$

Therefore, $P_{v}$ and $P_{t}$ are proportional to the vector and tensor polarizations of the $f=1$ state, respectively, while $P_{\Delta}$ indicates the deviation of the $f=0$ population density from its statistical factor $1 / 4$. Because of the small energy splitting between the $f=0$ and $f=1$ hyperfine states $(1.5 \mathrm{eV})$ compared to the $\mu^{-3}$ He binding energy, and hence small deviation of $P\left(f, f_{z}\right)$ from its statistical value, direct measurements of the angular correlation parameters are rather difficult 25, 26, 28].

The results reported here have been obtained using either the AV18 and AV18/UIX interactions or the older Argonne $v_{14}$ (AV14) two-nucleon interaction 29] in conjunction with the the Tucson-Melbourne (TM) three-nucleon interaction [30]. Note that both three-nucleon interactions have been adjusted to reproduce the triton binding energy.

Particular care has been put in the calculation of the $1 s$ wave function $\psi_{1 s}(x)$ of the muon in the electric field of the ${ }^{3} \mathrm{He}$ nucleus. Since the Bohr radius of the muonic atom is about $130 \mathrm{fm}$, i.e. much larger than the nuclear radius, it is well justified to approximate $\psi_{1 s}(x)$ in the computation of the weak transition matrix elements with an average value $\psi_{1 s}^{\text {av }}$, defined as:

$$
\psi_{1 s}^{\mathrm{av}}=\frac{\int d \mathbf{x} \mathrm{e}^{\mathrm{iq} \cdot \mathbf{x}} \psi_{1 s}(x) \rho(x)}{\int d \mathbf{x} \mathrm{e}^{\mathrm{iq} \cdot \mathbf{x}} \rho(x)},
$$

where $\rho(x)$ is the ${ }^{3}$ He nucleus charge distribution and $\mathbf{q}$ is the leptonic momentum transfer. Finally, it is common to introduce the factor $\mathcal{R}$ defined as [10, 11:

$$
\left|\psi_{1 s}^{\mathrm{av}}\right|^{2} \equiv \mathcal{R}\left|\psi_{1 s}(0)\right|^{2}=\mathcal{R} \frac{\left(2 \alpha m_{r}\right)^{3}}{\pi}
$$

where $\psi_{1 s}(0)$ denotes the Bohr wave function evaluated at the origin for a point charge $2 e$, and $m_{r}$ is the reduced mass of the $\mu^{-3}$ He system. The factor $\mathcal{R}$ has been calculated with the following procedure: for a given Hamiltonian model we have calculated $\rho(x)$. Knowing $\rho(x)$, the Poisson equation has been solved to obtain the static electric potential. Finally we have solved the Dirac equation and found $\psi_{1 s}(x)$. Using Eqs. (8) and (9), we have calculated $\mathcal{R}$ for the three nuclear Hamiltonian models considered here. The results are given in Table 11. They are in rather good agreement with the values given in literature [26].

Results for the capture rate $\Gamma_{0}$ and angular correlation parameters $A_{v}$, $A_{t}$, and $A_{\Delta}$ are presented in Table 2. The uncertainty (in parentheses) in the predicted values is due to the uncertainty in the determination of the $N \Delta$ 
Table 1. Values for $\mathcal{R}$ as defined in Eq. (9) for three different Hamiltonian models $H$.

\begin{tabular}{cc}
\hline$H$ & $\mathcal{R}$ \\
\hline AV18 & 0.979 \\
AV18/UIX & 0.980 \\
AV14/TM & 0.980 \\
\hline
\end{tabular}

transition parameter $g_{A}^{*}$, as discussed in the Introduction. The latter reflects the experimental error in the Gamow-Teller matrix element of tritium $\beta$-decay.

Inspection of Table 2 shows that the theoretical determination of the total capture rate $\Gamma_{0}$, when the AV18/UIX and AV14/TM Hamiltonian models are used, is within $1 \%$ of the recent experimental result [31]. Furthermore, the model dependence in the calculated observables is very weak: the AV18/UIX and AV14/TM results differ by less than $0.5 \%$. The agreement between theory and experiment and the weak model dependence mentioned above reflect, to a large extent, the fact that both the AV18/UIX and AV14/TM Hamiltonian models reproduce: i) the experimental binding energies as well as the charge and magnetic radii 32 of the three-nucleon systems; ii) the Gamow-Teller matrix element in tritium $\beta$-decay. In this respect, it is interesting to note that the capture rate predicted by the AV18 Hamiltonian model is about $4 \%$ smaller than the experimental value. The same result has been found with the two-nucleon AV14 Hamiltonian model [8].

The value for the angular correlation parameter $A_{v}$ listed in Table 2 is also in reasonable agreement with the corresponding experimental result which has however a rather large error. Note that the polarization observables are not sensitive to the inclusion of the three-nucleon force, as can be seen comparing the AV18 and AV18/UIX lines of Table 2.

The contributions of the different components of the weak current and

Table 2. Capture rate $\Gamma_{0}\left(\mathrm{sec}^{-1}\right)$ and angular correlation parameters $A_{v}, A_{t}$, and $A_{\Delta}$ calculated using PHH wave functions corresponding to the AV18, AV18/UIX and AV14/TM Hamiltonian models are compared with the experimental results. The theoretical uncertainties, shown in parentheses, reflect the uncertainty in the determination of the $N \Delta$ transition axial coupling constant $g_{A}^{*}$. The experimental values of $\Gamma_{0}$ and $A_{v}$ have been taken from Ref. 31] and [28], respectively. Here, we have assumed $R_{P S}=1$ in Eq. (2).

\begin{tabular}{crrrc}
\hline Observable & AV18 & AV18/UIX & AV14/TM & Expt. \\
\hline$\Gamma_{0}$ & $1441(7)$ & $1484(8)$ & $1486(8)$ & $1496(4)$ \\
$A_{v}$ & $0.5341(14)$ & $0.5350(14)$ & $0.5336(14)$ & $0.63(15)$ \\
$A_{t}$ & $-0.3642(9)$ & $-0.3650(9)$ & $-0.3659(9)$ & \\
$A_{\Delta}$ & $-0.1017(16)$ & $-0.1000(16)$ & $-0.1005(17)$ & \\
\hline
\end{tabular}


Table 3. Effects of the inclusion of the two-body currents for the muon capture rate $\Gamma_{0}\left(\right.$ in $\mathrm{sec}^{-1}$ ) and angular correlation parameters $A_{v}, A_{t}$, and $A_{\Delta}$. The PHH wave functions are obtained using the AV18/UIX Hamiltonian model. The column labeled "One-body" lists the contributions associated with the one-body vector and axial charge and current operators. The column labeled "Mesonic" lists the results obtained by including, in addition, the contributions from meson-exchange mechanisms. Finally the column labeled " $\Delta$ " lists the results obtained by including also the $\Delta$-excitation contributions. The experimental vaues of $\Gamma_{0}$ and $A_{v}$ are taken from Ref. 31] and [28], respectively. Here, we have assumed $R_{P S}=1$ in Eq. (2).

\begin{tabular}{ccccc}
\hline Observable & One-body & Mesonic & $\Delta$ & Expt. \\
\hline$\Gamma_{0}$ & 1316 & 1384 & 1484 & $1496(4)$ \\
$A_{v}$ & 0.5749 & 0.5511 & 0.5350 & $0.63(15)$ \\
$A_{t}$ & -0.3565 & -0.3679 & -0.3650 & \\
$A_{\Delta}$ & -0.0686 & -0.0810 & -0.1000 & \\
\hline
\end{tabular}

charge operators to the observables are reported for the AV18/UIX model in Table 3. The column labeled "One-body" lists the contributions associated with the one-body terms of the vector and axial charge and current operators, including relativistic corrections proportional to $1 / \mathrm{m}^{2}$. The column labeled "Mesonic" lists the results obtained by including, in addition, the contributions from two-body vector and axial charge and current operators, associated with pion- and vector-meson-exchanges. Finally, the column labeled " $\Delta$ " lists the values of the observables obtained by including the contributions arising from $\Delta$ excitation.

Among the observables, $\Gamma_{0}$ and $A_{\Delta}$ are the most sensitive to two-body contributions in the weak current. These are in fact crucial for reproducing the experimental capture rate, see Table 3 .

An important motivation of the present work is to test the sensitivity of the muon capture observables to the induced pseudo-scalar form factor $G_{P S}$ and, eventually, infer its value from the $\Gamma_{0}$ measurement. Therefore, we have repeated the calculation using AV18/UIX PHH wave functions and several different values of the parameter $R_{P S}$, defined in Eq. (2). The variation of each observable in terms of $R_{P S}$ is displayed in Fig. 1 . The angular correlation parameters, in particular $A_{t}$ and $A_{\Delta}$, are more sensitive to changes in $R_{P S}$ than the total capture rate, as first pointed out in Ref. [27]. A precise measurement of these polarization observables could therefore be useful to ascertain the extent to which the induced pseudo-scalar form factors deviate from their PCAC values.

By enforcing perfect agreement between the experimental and theoretical values, taken with their uncertainties, for the total capture rate $\Gamma_{0}$, it is possible to obtain an estimate for the range of values allowed for $R_{P S}$, and we have found

$$
R_{P S}=0.94 \pm 0.06
$$




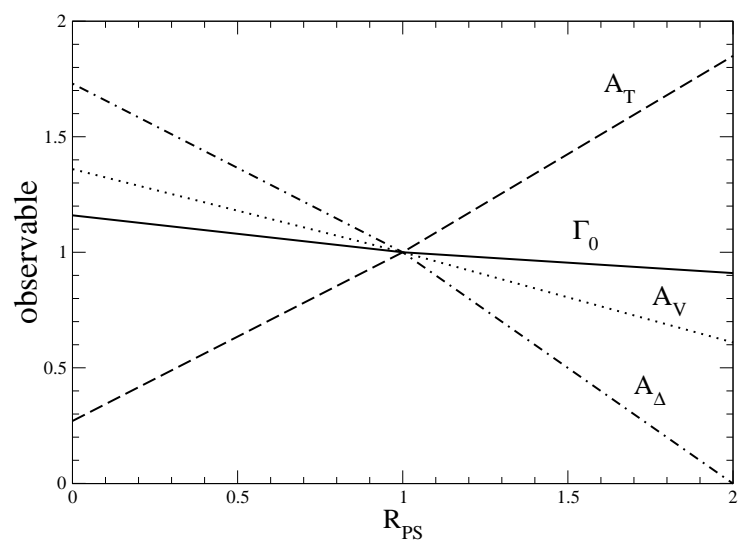

Figure 4. Variation of the capture rate $\Gamma_{0}$ and angular correlation parameters $A_{v}$, $A_{t}$, and $A_{\Delta}$ with the parameter $R_{P S}$ entering the expression for the induced pseudoscalar coupling $G_{P S}$ given in Eq. (2). The AV18/UIX PHH wave functions are used. For each observable, the ratio between the result obtained with the given value of $R_{P S}$ and that one with the PCAC value $R_{P S}=1$ is plotted as function of $R_{P S}$.

This $6 \%$ uncertainty is significantly smaller than that found in previous studies [26, 33, 34]. This substantial reduction in uncertainty can be traced back to the procedure used to constrain the (model-dependent) two-body axial currents discussed in the Introduction.

\section{Summary and Outlook}

We have reported new calculations of $p-d$ radiative capture observables at energies above the DBT, and of observables in the process ${ }^{3} \mathrm{He}\left(\mu^{-}, \nu_{\mu}\right)^{3} \mathrm{H}$. These calculations have been based on the Argonne $v_{18}$ two-nucleon and Urbana IX three-nucleon interactions. For the muon capture reaction also the older Argonne $v_{14}$ two-nucleon and Tucson-Melbourne three-nucleon interactions have been used. Accurate bound and continuum wave functions have been obtained with the PHH method. The model for the EM and weak transition operators has been taken to consist of one- and two-body components. In recent studies, this theory has been shown to correctly predict the static properties of the three-nucleon systems [32], as well as their associated elastic and transition electromagnetic form factors.

A satisfactory description of measured $p-d$ capture observables at $E_{\text {c.m. }}=$ $3.33 \mathrm{MeV}$ has emerged with the exception of the $T_{20}$ and $T_{21}$ tensor analyzing powers. Interestingly, the very same discrepancies were observed also below the DBT, namely at $E_{\text {c.m. }}=2 \mathrm{MeV}$. Similarly, at higher values of $E_{\text {c.m. }}$, differential cross sections and $A_{y}(d)$ seem to be well reproduced, while the tensor analyzing powers $A_{x x}, A_{y y}$ and $A_{z z}$ at $E_{\text {c.m. }}=5.83 \mathrm{MeV}$ present the same discrepancies mentioned above. 
In order to clarify the origin of these discrepancies, we plan to extend the calculation of $p-d$ capture observables at even higher energies, and to investigate alternative models for short-range two-body EM currents.

In regard to the muon capture process, we have found that the predicted total rate is in agreement with the experimental value, and has only a weak model dependence: the AV18/UIX and AV14/TM results differ by less than $0.5 \%$. As discussed above, the weak model dependence comes about because both Hamiltonians reproduce the binding energies, charge and magnetic radii of the three-nucleon systems, and the Gamow-Teller matrix element in tritium $\beta$-decay.

It is important to note that, if the contributions associated with two-body terms in the axial current were to be neglected, the predicted capture rate would be 1316 (1318) $\mathrm{sec}^{-1}$ with AV18/UIX (AV14/TM), and so two-body mechanisms are crucial for reproducing the experimental value. The present work shows that the procedure adopted for constraining these two-body contributions leads to a consistent description of available experimental data on weak transitions in the three-body systems. It also corroborates the robustness of our recent predictions for the cross sections of the proton weak captures on ${ }^{1} \mathrm{H} \mathrm{[15]}$ and ${ }^{3} \mathrm{He}[7]$, which were obtained with the same model for the nuclear weak current.

Finally, it would be interesting to extend our investigation to the ${ }^{3} \mathrm{He}\left(\mu^{-}, \nu_{\mu}\right) n d$ and ${ }^{3} \mathrm{He}\left(\mu^{-}, \nu_{\mu}\right) n n p$ processes, both of which have been studied experimentally in Ref. [35] and theoretically in Ref. [36]. Work along these lines is vigorously being pursued.

The work of R.S. is supported by the U.S. Department of Energy contract number DE-AC05-84ER40150 under which the Southeastern Universities Research Association (SURA) operates the Thomas Jefferson National Accelerator Facility.

\section{References}

1. A. Kievsky et al.: Phys. Rev. C58, 3085 (1998)

2. W. Glöckle et al.: Phys. Rep. 274, 107 (1996)

3. A. Kievsky et al.: Nucl. Phys. A607, 402 (1996)

4. R. Schiavilla et al.: Phys. Rev. C40, 2294 (1989)

5. R. Schiavilla and D. O. Riska: Phys. Rev. C43, 437 (1991)

6. J. Carlson and R. Schiavilla: Rev. Mod. Phys. 70, 743 (1998)

7. L.E. Marcucci et al.: Phys. Rev. Lett. 84, 5959 (2000); Phys. Rev. C63, 015801 (2001)

8. L.E. Marcucci et al.: Phys. Rev. C66, 054003 (2002)

9. T.R. Hemmert et al.: Phys. Rev. D51, 158 (1995) 
10. J.D. Walecka: In: Muon Physics; Weak Interactions, edited by V.W. Hughes and C.S. Wu, p. 114. New York: Academic Press 1975

11. J.D. Walecka: Theoretical Nuclear and Subnuclear Physics New York: Oxford University Press 1995

12. D.O. Riska: Phys. Scr. 31, 471 (1985); A. Buchmann et al.: Nucl. Phys. A443, 726 (1985)

13. R. Schiavilla et al.: Phys. Rev. C45, 2628 (1992)

14. M. Kirchbach et al.: Nucl. Phys. A542, 616 (1992)

15. R. Schiavilla et al.: Phys. Rev. C58, 1263 (1998)

16. A. Kievsky et al.: Nucl. Phys. A551, 241 (1993); Nucl. Phys. A577, 511 (1994)

17. M. Viviani et al.: Phys. Rev. C54, 534 (1996)

18. M. Viviani et al.: Phys. Rev. C61, 064001 (2000)

19. A. Kievsky et al.: Phys. Rev. C64, 024002 (2001)

20. F. Goeckner et al.: Phys. Rev. C45, R2536 (1992)

21. B.D. Belt et al.: Phys. Rev. Lett. 20, 1120 (1970)

22. H. Akiyoshi et al.: Phys. Rev. C64, 034001 (2001)

23. R.B. Wiringa et al.: Phys. Rev. C51, 38 (1995)

24. B.S. Pudliner et al.: Phys. Rev. Lett. 74, 4396 (1995)

25. D.F. Measday: Phys. Rep. 354, 243 (2001)

26. J.G. Congleton and H.W. Fearing: Nucl. Phys. A552, 534 (1992)

27. W.-Y.P. Hwang: Phys. Rev. C17, 1799 (1978)

28. P.A. Souder et al.: Nucl. Instr. and Meth. in Phys. Res. A402, 311 (1998)

29. R.B. Wiringa et al.: Phys. Rev. C29, 1207 (1984)

30. S.A. Coon et al.: Nucl. Phys. A317, 242 (1979)

31. P. Ackerbauer et al.: Phys. Lett. B417, 224 (1998)

32. L.E. Marcucci et al.: Phys. Rev. C58, 3069 (1998)

33. J.G. Congleton and E. Truhlik: Phys. Rev. C53, 956 (1995)

34. J. Govaerts and J.-L. Lucio-Martinez: Nucl. Phys. A678, 110 (2000)

35. S.E. Kuhn et al.: Phys. Rev. C50, 1771 (1994)

36. R. Skibinski et al.: Phys. Rev. C59, 2384 (1999) 